\title{
Réflexivité herméneutique de la créativité en traduction
}

\section{Hermeneutic Reflexivity of Creativity in Translation}

\author{
Jacob Cléophas DEFO NZIKOU ${ }^{1}$
}

${ }^{1}$ Postgraduate Student, University of Dschang, Arts and Social Sciences, Dschang, Cameroon

ORCID: J.C.D.N. 0000-0002-8342-2270

\section{Corresponding Author:}

Jacob Cléophas DEFO NZIKOU, University of Dschang, Arts and Social Sciences, Dschang, Cameroon

E-mail: jacob.defo@gmail.com

\section{Submitted: 18.02 .2020}

Revision Requested: 31.05 .2020 Last Revision Received: 01.06.2020 Accepted: 01.07.2020

Citation: Defo Nzikou, J. C. (2020). Réflexivité herméneutique de la créativité en traduction. Litera, 30(2), 753-777. https://doi.org/10.26650/LITERA2020-0043

\begin{abstract}
RÉSUMÉ
Les études en traduction au sujet du dilemme qu'elle suscite sont devenues encore plus pertinentes à une époque où le terme "créativité" y a été introduit pour exprimer une meilleure compréhension de ce qui fut d'antan perçu comme de la "trahison". La recrudescence du débat, ces dernières années, au tour de l'alternative fidélité versus créativité, montre que nous n'avons pas pour autant été dissuadé du fait de rompre avec le vœu absolu de fidélité qui maintenait la traduction dans un statut dilemmatique. Nous situant du point de vue d'une expertise herméneutique de la traduction, cet article tente de soutenir que la créativité n'est pas une simple alternative, mais une nécessité qui s'impose en situation. Pour étayer ce point, l'analyse de la réflexivité herméneutique de la créativité en traduction repose sur le caractère interprétatif du traduire qui met en jeu le phénomène de la compréhension. Partant de la mise en exergue de la fécondité de l'acte, nous démontrons qu'elle justifie amplement le fait qu'une traduction soit équivalente. II s'agit au fond, de démontrer que la traduction est construction d'équivalence de sens et de valeur. L'article conclut que, de manière cohérente avec le fait que la créativité n'est pas une alternative, le vœu de fidélité participe de la dialectique de l'émergence du sens et conforte l'idée selon laquelle la traduction est un projet créatif.
\end{abstract}

Mots-clés: Réflexivité, créativité, fidélité, traduction, herméneutique

\section{ABSTRACT}

Translation studies on this dilemma have become even more relevant at a time when the term "creativity" has been introduced to express a better understanding of what was once perceived as "betrayal". The resurgence in recent years of the debate on faithfulness versus the creativity alternative, shows that we have not been dissuaded from breaking with the absolute vow of faithfulness which has kept translation in a dilemmatic status. From the point of view of hermeneutic expertise, this article attempts to argue that creativity is not a simple alternative, but a necessity which is essential when translating. To support this point, the analysis of the hermeneutic reflexivity of creativity in translation is based on the interpretative character of translating which brings into play the phenomenon of understanding. Starting from highlighting the fertility of the act, we demonstrate that it amply justifies the fact that a translation is equivalent. It is basically a question of demonstrating that translation is the construction of an equivalence of meaning and value. The article concludes that, in a manner consistent with the fact that creativity is not an alternative, the vow of faithfulness participates in the dialectic of the emergence of meaning and supports the idea that translation is a creative project.

Keywords: Reflexivity, creativity, faithfulness, translation, hermeneutics 


\section{EXTENDED ABSTRACT}

Translation studies on this dilemma have become even more relevant at a time when the term «creativity» has been introduced to express a better understanding of what was once perceived as «betrayal». The resurgence in recent years of the debate on faithfulness versus the creativity alternative, shows that we have not been dissuaded from breaking with the absolute vow of faithfulness which has kept translation in a dilemmatic status. Generally speaking, academic perspectives on the question revolve around several notions that result from this dilemmatic status. The most recurrent are the concepts of equivalence and adequacy. These notions most often indicate divergent views which find their resonance at the level of practice. We therefore have the impression that there is a theoretical instability in translation if we refer to these two trends. The first considers textual faithfulness as an absolute wish, which aims to «instil in the translated work the spirit of a language which is foreign to it» (Schleiermacher, 1999). The second trend focuses on the power of action of the translator who, in the process of interpretative negotiation, engages in a creative project. Looking closely, through the prism of hermeneutics, we think that the concept of creativity somehow comes to assert a theoretical stability in this process.

In this theoretical landscape, the appearance of the concept of creativity seems to us to represent a turning point, because it characterizes the very essence of translation. We demonstrate this by referring to the hermeneutic breeding ground which allows us to see creativity no longer as an alternative, but a necessity which is essential when translating and which constitutes the very being of translation. By relying on the interpretative character of translating, we account for an intrinsic fruitfulness which materializes by the fact that we can always say it a different way. This is explained firstly by the fact that having a closure between the idioms means that translating consists of a production rather than a reproduction. Secondly, because the translator-interpreter only redeploys what makes sense according to his own understanding of the language. This implies a possibility of saying more in the face of the ineffable, thus exceeding what has been proposed. «Translation, like all interpretation, is a highlighting. A translator must understand that highlighting is part of his task» (Gadamer, 1996, p. 408). Thus, the restitution is creative, which side-lines any vow of textual faithfulness. What matters is coming to a proper understanding about the subject matter. As soon as there is an understanding, each sign is interpreted from its potential: meaningfulness. This reflects an ability to mean more. At the end of the day, the creative gesture which supposes it, 
consists of the search for equivalences of meaning. Creativity in translation relates to the idea that «a good translation can only aim for an alleged equivalence that is unfounded in an identity of demonstrable meaning» (Ricœur, 2004, p. 40).

However, we cannot ignore the question of limits or the infinity of interpretation. We cannot deny that the interpretation for more meaning could contribute to an abuse of meaningfulness. The creativity that prevails here can be seen as the effect of a refusal to impregnate the culture of the source language. Also, some authors, like Eco, think that «we must resist the temptation to help the text too much, by almost replacing the author» (Eco, 2006, p. 126). According to this author, for example, «a translation that can» say more «may be an excellent work in itself, but not a good translation» (Eco, 2006, p. 139). In any case, we believe that the value of a good translation is judged by the effectiveness of the meaning rendered - which a creative translation can do easily.

The article concludes that the vow of faithfulness participates in the dialectic of the emergence of meaning and supports the idea that translation is a creative project. This project seems to have mobilized elements attributable both to the «reception aesthetic» and to «language hospitality» for its effectiveness. Consistent with the fact that creativity is not an alternative, we can say that any translation aims for an equivalence of meaning and value between the two statements following an interpretative negotiation between the translator and the text. 


\section{Introduction}

Ce qui est propre doit être aussi bien appris que ce qui est étranger.' Mais alors, pourquoi ce désir de traduire doit-il être payé du prix d'un dilemme, le dilemme fidélité/trahison ? (Ricœur, 2004, p. 39)

En mettant en exergue cette citation, nous nous sommes posé la question suivante : Serait-il superflu de nous demander quelle serait aujourd'hui la nécessité d'une réflexion sur le dilemme du traduire ? II ne nous semble pas sans intérêt, car la question est partagée par des décennies de recherches fructueuses sur la traduction. Selon Ricœur les raisons d'une telle productivité au tour de ce dilemme consistent, par exemple, dans le fait qu'il n'existe pas de critère absolu de bonne traduction (Ricœur 2004, p. 39). Quoiqu'il puisse être, on note tout de même une progression en terme d'acquis sur la question. Ceci se reflète dans la façon dont on aborde le sujet aujourd'hui. De fait, on peut remarquer que les formulations thématiques se passent de plus en plus d'une tournure qui vise à stigmatiser l'acte de traduire, à l'instar de la formulation : fidélité versus trahison. Mais il reste que l'on demeure dans l'idée d'une alternative : fidélité versus créativité. Quoique cette nouvelle alternative qui substitue l'ancienne, témoigne d'une réalité indéniable au sein de la pratique de cet acte. Elle tire la sonnette d'alarme sur le fait qu'une créativité ou qu'un manque de fidélité textuelle est en lui-même un thème extrêmement riche qui se situe à la croisée de l'esthétique de la réception (Jauss, 1978) et de l'hospitalité langagière (Ricœur, 2004) dont la réflexivité herméneutique pourrait ici nous aider à mieux saisir le sens. En outre, il nous semble s'agir d'un phénomène croissant dans les sciences humaines où la traduction sert toujours de jonction ou d'instrument pour la réception et la promotion de connaissances.

Cependant, malgré de nombreuses recherches prolifiques au tour de la traduction qui explicite la nécessité ou non d'une créativité par des théories multiformes, l'on semble ignorer le vivier herméneutique qui serait le plus à même de décliner le phénomène qui se produit au cour de cet acte et donc la méconnaissance contribuerait à considérer la créativité inhérente comme une simple alternative. La référence à ce vivier se justifie par le fait que la traduction suppose au préalable une «traduction à l'intérieur de la même communauté langagière» (Ricœur, 2004, p. 43) que l'on qualifie de traduction intralinguistique - au sens ou «comprendre, c'est traduire» (Steiner, 1998) 
- qui, à la vérité, est un acte herméneutique. Premièrement parce que hermeneuein/ herméneuô signifie aussi «traduire» et deuxièmement parce que la traduction est en soi un «cas extrême» de l'acte herméneutique, si l'on considère que tout traducteur est interprète (Gadamer, 1996, pp. 407-409). Sur ces entrefaites, cet article essaye de montrer, au moins très succinctement, le sens de cette nouvelle expression qu'est la créativité à l'effet de proposer un encadrement aux stratégies du traducteur.

En général, ces perspectives peuvent permettre de faire le point sur deux approches: la traduction adéquate et la traduction équivalente. La première, la traduction adéquate «est celle qui actualise en langue cible les relations textuelles d'un texte original sans pour autant enfreindre son système linguistique» (Toury, 2004, p. 98). Par contre, la seconde, la traduction équivalente est liée au résultat de l'action traductionnelle, c'est-à-dire au rapport de valeur communicationnelle égal entre deux textes. Il est donc question d'éclairer, à partir de la perspective herméneutique, sur le fait que la notion de créativité ne nous plonge pas dans un nouveau dilemme, mais qu'elle est en elle-même l'essence de la traduction. L'indétermination apparente ici serait due à la dialectique de l'identité et de la différence, du désir de fidélité et du désir de satisfaction du lecteur. Étant donné qu'il faut consentir à la perte de quelque chose (Ricœur, 2004, p. 8) nous pensons que, tant que la fidélité au sens n'est pas menacée, la traduction est essentiellement créative parce qu'intrinsèquement féconde. Or, cette noble conviction peut être remise en question par la thèse selon laquelle la tâche du traducteur consisterait en une reproduction fidèle de l'œuvre originale et non en la création d'une œuvre nouvelle. D'où le problème de la valeur d'une traduction. Qu'estce qui fait la valeur d'une traduction ? Est-ce la conformité langagière ? Ou la capacité de rendre le sens?

Ce que nous souhaitons faire dans le cours de cet article, c'est montrer comment la créativité que nous jugeons intrinsèque au traduire est tributaire du noble désir de traduire. Nous proposons de mener notre analyse en trois étapes. Dans un premier temps nous voulons rendre compte de la fécondité intrinsèque de la traduction qui justifierait le fait consensuel d'une créativité. Ceci nous amènera à montrer comment d'un point de vue pratique elle est une équivalence de sens et de valeur entre les deux textes. Cela nous conduira finalement à statuer sur l'élément consensuel en traduction au regard du caractère toujours problématique d'une détermination d'une théorie de la traduction. 


\section{Fécondité intrinsèque au traduire : à l'épreuve de la créativité}

L'initiative de traduire qui consiste dans un double acte d'interprétation et de transposition de sens, se fait dans une intention de fidélité. Sauf que, cette pratique est toujours stigmatisée comme étant toujours déjà un acte de trahison : traduttore, traditore. Toutefois, nous nous rendons compte que l'apparente trahison serait tributaire, non seulement du fait que le texte d'arrivée n'est pas l'original, mais aussi du fait que l'acte même de traduire qui se caractérise par un phénomène de changement de sphère linguistique, suppose un changement d'idiome. Ceci étant, la créativité se présente comme étant le concept caractérise ce qui était perçu comme de la trahison. À la vérité, ce qu'il faudrait comprendre c'est que la traduction est intrinsèquement féconde. C'est pourquoi, bien qu'il y ait une recrudescence d'une réclamation de la fidélité textuelle dès qu'il s'agit du traduire, la plupart des essayistes reconnaissent qu'il s'agit d'un idéal dont l'exigence de l'atteindre se trouve aussitôt vacillée dès qu'on se livre à l'acte. De fait, on ne peut plus ignorer les risques d'un vœu absolu de fidélité textuelle, car la traduction littérale que cela suppose expose à une possibilité de perte de sens.

En effet, les premières théories qui se focalisaient sur la traduction littérale se sont concentrées sur l'aspect idiomatique des langues pour conclure qu'elles étaient intraduisibles du fait de leurs hétérogénéités radicales. La question de la possibilité de traduire qui se posa ici demeura spéculative et sera qualifiée d'«alternative ruineuse» (Ricœur, 2005, p. 25), car au regard des preuves empiriques de traductions, on ne put nier le fait qu'on puisse tout de même traduire. Le fait est qu'«en dépit de l'hétérogénéité des idiomes, il y a des bilingues, des polyglottes, des interprètes et des traducteurs» (Ricœur, 2004, p. 33) qui témoignent de la réalité de la traduction. Toutefois, étant donné que celle-ci n'était pas toujours littérale et donc fidèle, il fut facile de stigmatiser l'acte par le concept de trahison.

Nonobstant, il s'est avéré que l'apparente trahison est l'effet d'une fécondité intrinsèque à tout acte de traduire qui fait d'une certaine fidélité radicale un leurre. Puisque,

Par la traduction, le travail de la pensée se trouve transposé dans l'esprit d'une autre langue, et subit ainsi une transformation inévitable. Mais cette transformation peut devenir féconde, car elle fait apparaître en une lumière nouvelle la position fondamentale de la question ; elle fournit 
ainsi l'occasion de devenir soi-même plus clairvoyant et d'en discerner plus nettement les limites. (Heidegger, 1937, p. 11)

Traduire consisterait à extraire la pensée de cette gangue idiomatique propre à la langue source pour l'insérer dans celle de la langue cible. Ce qui laisse une part de choix et de liberté au traducteur dans son appropriation de l'idiome de la langue cible. Il est question d'opérer une «migration linguistique» (Ladmiral, 1994, p. 15). Cependant, il est à noter que ce qui migre n'est pas la langue, mais le discours. En outre, ce qui migre ne saurait être la façon dont le discours est tissé dans la langue source. Donc, l'acte de traduire consiste à redire ceux dont il est question dans le texte, dans l'autre langue, comme cela a été fait dans la langue source, mais pas forcément comme dans la langue source.

Il ne s'agit pas au fond d'imitation ou de reproduction, mais plus de production. C'est le fait de devoir produire qui fait dire qu'il y a créativité. Nous l'aurions compris, cette «infidélité textuelle» n'est pas un acte malsain. Parce que, la manière par laquelle le traducteur essaye de signifier, dans la langue hôte, dépend des propriétés linguistiques de cette langue qui ne sont pas toujours conformes à la langue de départ. Toute l'entreprise consiste ici à éviter une traduction littérale qui concourrait à la perte de sens. Ainsi, on peut relativiser le sens de la fidélité, car le traducteur ne devrait s'engager qu'à une loyauté vis-à-vis du texte, et non de la langue. La fidélité textuelle, supposant le mot à mot ou le mot pour mot, pourrait créer un contre sens ou un non-sens. C'est pourquoi, ce que devrait faire, à la vérité le traducteur, c'est extraire le sens des mots pour le recouvrir des mots, savamment choisit, de l'autre langue. Puisque,

Si l'on cherche consciencieusement une équivalence entre des mots, quand les hispanophones disent mesita de luz (petite table de «lumière»), on trouve que les Français et les Anglais disent table de nuit ou night-table. Tandis qu'un Anglais prend des photos, I'Espagnol les tire et le Guarani les enlève. Les Français et les Espagnols présentent leurs amis mais les Anglais les introduisent (to introduce). Les choses arrivent en français, (happen) en anglais et passent (pasan) en espagnol. [...] En espagnol quand on rencontre quelqu'un, on lui dit «cómo te va ?» («comment te va»?), en français on lui demande comment vas-tu ? et en anglais how are you ? (comment es-tu ?) ou how do you do ? (comment fais-tu ? ou comment le fais-tu?). (Díez, 2014, pp. 89-90) 
Cette illustration de Díez permet de nous rendre compte que la correspondance n'est pas donnée de façon littérale, mais construite. Cette construction se fait au niveau du « sens ». Donc, la créativité lors de la traduction est tout à fait commode lorsqu'il s'agit d'une réexpression d'une existence de la réalité dans une autre langue.

D'un point de vue herméneutique, tout ceci n'est pas dénué de sens, car l'acte de traduire est un acte interprétatif. De plus, le caractère langagier de la scène nous convie à mieux saisir le phénomène de la traduction de par sa réflexivité herméneutique. En vérité, qui dit langage, dit événement de sens et non instrument de désignation et de communication de ce qui a déjà été pensé. Aussi, l'interprète usant d'un langage, ne peut que redéployer ce qui fait sens. Ce qui implique une possibilité de dire davantage face à l'ineffable, excédant ainsi la parole proposée. Cette interprétation est créative, puisqu'en tant qu'acte herméneutique, elle s'intéresse au sens de la chose du texte qu'elle redéploye autrement.

Le geste herméneutique qui opérera la rencontre de l'interprète et de la chose sera logiquement pensé à l'aide de schémas spatiaux, ayant tous une connotation commune de propriété, de territorialité, de demeure, d'«habiter». [...] Interpréter revient à ouvrir le texte ou la pratique, à ravir, à l'un et à l'autre, leur secret, pour les insérer dans la dimension symbolique occupée par le lecteur. [...] L'interprétation exprime l'événement même d'ouverture du logos qui [...] est déploiement, dépliage, expansion et extension symboliques. (Resweber, 1988, pp. 24-25)

L'ouverture du logos par extension symbolique implique un surcroît d'être qui est tributaire d'un retour à la chose du texte lors de l'interprétation. C'est ce surcroît qui se présente comme l'autrement et qui témoigne du fait d'avoir pensé de nouveau cette chose dans l'autre langue par un processus de restitution créative.

Ainsi, traduire c'est interpréter, «car tout traducteur est interprète» (Gadamer, 1996, p. 409). Aussi Resweber pense que l'interprète en tant qu'observateur et auditeur du sens, va de la lecture à la relecture, car le sens, une fois lu, découvert et recueilli, doit être relu, rassemblé ou réintégré, pour faire corps (Resweber, 1988, pp. 26-31). Ce qui l'amène à conclure que «le traducteur, en entendant une langue dans une autre langue, transcrit cette écoute dans des jeux de langage qui ne manquent pas de déplacer les significations propres à chaque langue» (Resweber, 1988, p. 123). La traduction dans cette condition 
interprétative ne peut s'envisager comme une opération devant souscrire absolument à la fidélité textuelle. Elle tient compte du fait que le texte de la langue originaire est un discours ; «chaque traduction engage [...] une interprétation du discours en question» (Michon, 2000, p. 47). Aussi, l'interprétation consistera en l'acquisition du sens.

Si nous présumons que ce que l'on comprend au cours d'une interprétation n'est autre que le sens, nous admettrions que le passage d'une langue à l'autre dans le processus de la traduction consiste en la transposition du sens issu de l'interprétation.

Le traducteur a pour tâche de transposer le sens à comprendre dans le contexte où vit l'interlocuteur. Bien entendu, cela ne veut pas dire que le traducteur soit autorisé à trahir le sens que l'autre a en vue. Ce sens doit être, au contraire, conservé ; mais, comme il doit être compris dans le monde d'une autre langue, il est nécessairement mis en vigueur d'une autre manière. C'est pourquoi toute traduction est déjà interprétation (Auslegung) ; on peut même dire qu'elle est toujours l'accomplissement de l'interprétation que le traducteur a donnée de la parole qui lui a été proposée. (Gadamer, 1996, p. 406)

Gadamer fait état ici, d'une nécessité qui s'impose, au cours du transfert du sens. C'est celle de devoir l'exprimer d'une autre manière, c'est-à-dire en y mettant du sien par un acte créatif sans toutefois trahir le sens. Cependant, on peut se demander comment est-il tout de même possible de faire vivre le sens d'une «autre manière» dans l'autre langue sans que l'acte créatrice ne soit préjudiciable.

À la vérité, la compréhension du sens est inséparable de la conception de son origine ou de sa source (Marc de Launay, 2006, p. 73 sq). En outre, la traduction consiste en une reprise guidée par la compréhension de ce qui est dit (Gadamer, 1996, p. 407). Conscient de ceci, le traducteur ayant su ce qui est dit, a pour tâche de le rendre dans l'autre langue. Toutefois, le langage étant le milieu dans lequel se réalise l'entente entre l'auteur et le lecteur-traducteur, l'accord sur la chose même du texte serait une raison suffisante pour le traducteur de le redéployer à son aise. Il est question pour le traducteur qui se réfère à l'élément langagier de s'approprier le sens sans se priver de tous ensemble signifiant que lui propose son interprétation et de le repenser dans l'autre langue. Gadamer est tout à fait fondé d'y voir un problème herméneutique axé sur la qualité de l'entente sur la chose du texte. Il écrit : 
Le problème herméneutique est donc celui que pose, non pas la véritable maîtrise de la langue, mais la qualité de l'entente sur quelque chose, à laquelle on parvient dans ce milieu qu'est la langue. Toute langue peut être apprise au point qu'en la possédant à la perfection on ne traduise plus de ou dans la langue maternelle, mais que l'on pense dans la langue étrangère. (Gadamer, 1996, p. 407)

C'est le fait de penser dans l'autre langue qui est malheureusement perçu comme un acte d'infidélité textuelle. II faut noter que, la conscience d'une différence des idiomes d'une langue à l'autre dès le vouloir traduire n'enlise en rien le désir d'une fidélité textuelle au relent de la littéralité chez le traducteur. Cet inconfort psychologique amène à se rendre compte que : «la question est double : 1/ Dois-je être littéral ? 2/ Peut-on être littéral ?» (Ladmiral, 2013, p. 22). Si on est littéral, la traduction risque d'être une translittération qui consiste à transcrire ou à co-opérer. Or, la tâche consiste à instituer ce qui est dit dans la langue étrangère, ce qui ne saurait être une réécriture. Étant donné la synergie conceptuelle spécifique de chaque langue, le traducteur est contraint de tenir compte de la globalité de chacune d'elle pour produire son discours, et ceux en fonction de son génie propre et de sa vision de la langue. Schleiermacher (1999) note d'ailleurs que

toute langue comporte, en dépit des diverses opinions simultanées ou successives, un système de concepts qui, précisément parce qu'ils sont en contact, se lient et se complètent dans la même langue, constituent un tout dont les composantes singulières ne correspondent à aucune de celles du système des autres langues, et c'est à peine si font exception Dieu et l'Être, le substantif et le verbe originaires. Car même ce qui est purement et simplement universel, et bien qu'il se situe hors du champ de la particularité, reçoit de la langue son éclairage et sa couleur. (Cité par Marc de Launay, 2006, pp. 102-103)

Il s'avère ici que l'éclairage tout comme la coloration particulière participent de la traduction et donc de sa dimension créative. L'essentiel étant le sens, la phase interprétative qui permet son acquisition est suivie par la phase d'institutionnalisation dans l'autre langue. Ce qui corrobore au paradoxe de la traduction tel qu'élucidé par Schleiermacher : «Amener le lecteur à l'auteur», «amener l'auteur au lecteur» (Ricœur, 2004 , p. 9, p. 16, p. 41). L'effectuation de ceci ne saurait être autrement que sous fond 
créatif, car la transmission ici suppose une explicitation rendant possible la saisie du dit dans la langue du lecteur, même par un éclairage nouveau. D’ailleurs,

C'est sous un éclairage nouveau, provenant de l'autre langue, que, pour celui qui le lit dans cette langue, le texte est placé. L'exigence de la fidélité imposée à la traduction ne peut supprimer la différence fondamentale des langues. Aussi fidèles que nous voulions être, nous sommes placés en face de décisions délicates. Quand dans notre traduction, nous voulons souligner un trait du texte original qui nous semble particulièrement important, nous ne pouvons le faire qu'aux dépends d'autres traits ou au prix de leur élimination. [...] Comme toute interprétation la traduction apporte un excès de clarté (Überhellung), ce dont tout traducteur doit prendre la responsabilité. (Gadamer, 1996, pp. 407-408)

Ainsi, toute traduction est une occasion de rendre clair ce qui était flou dans le texte de départ. Voilà pourquoi, Gadamer souligne que «toute traduction qui prend sa tâche au sérieux est aussi plus claire et plus plate que l'originale» (Gadamer, 1996, p. 408). Et si traduire, c'est rendre claire, elle suppose inéluctablement une part de créativité dans l'opération de translation. La liberté du traducteur à opérer un choix stylistique entraine, en effet, des pertes qu'il convient d'assumer dans l'entreprise de toute traduction. En traduction «il est procédé à un certain sauvetage et à un certain consentement à la perte» (Ricœur, 2004, p. 8); ce qui est sauvé c'est le message au détriment de l'idiome de la langue de départ. N'est pas dire qu'au bout du compte, on parvient à une équivalence sans adéquation?

\section{Créativité et traduction équivalente : une cohérence}

Notre examen précédent nous a permis de reconnaître le caractère inhérent de la créativité en traduction. Celle-ci s'avère être l'effet d'une autonomie de la langue, car le texte littéraire est un «type particulier de production de messages qui se définit par son autonomie et sa clôture» (Hénault, 1979, p. 185). Mais ceci ne voudrait pas dire que le message diffère d'un texte à l'autre. Il doit y avoir une correspondance entre les textes. Ce qui présume une certaine objectivité de la créativité. En effet, si nous reconnaissons que la traduction est «l'interprétation des signes d'une langue au moyen des signes d'une autre langue» (Mounin, 1986, p. 7), nous comprendrions qu'il n'est pas question d'escompter une adéquation ou une identité à l'issue de celle-ci. 
La créativité n'est pas ici un pis-aller, à défaut d'être littéral. Elle traduit un souci de perfection qui habite l'homme à chaque fois qu'il veut signifier quelque chose de façon langagière. Pour le comprendre, nous pouvons nous référer à Humboldt qui fait remarquer que «les langues sont le produit de la force de l'esprit humain. Partout où il y a langue, la force langagière originelle de l'esprit humain est à l'œuvre, et chaque langue a la capacité d'atteindre le but général que vise cette force naturelle de l'homme» (Gadamer, 1996, p. 463). Cette force se matérialise, en tout maniement de la langue, par une aspiration à la perfection langagière d'une existence dans la réalité. Ainsi, tout comme l'auteur du texte de départ exprime la chose du texte en aspirant à une perfection langagière, le traducteur y aspire aussi. II ne se comporte pas comme un scribe qui reproduit, il produit.

Le lecteur-traducteur lit d'abord le texte publié dans la langue de départ. Avant de le traduire, il essaie de construire un monde imaginaire, parfois différent de celui de l'auteur propre de ce texte. À la fin de sa construction, il essaie de traduire, de transformer son monde imaginaire dans une autre culture. Par conséquent, il est d'abord un consommateur (du texte produit par l'auteur dans la langue de départ), puis producteur du monde imaginé par l'auteur et ensuite un créateur (traducteur) de ce monde imaginé dans une autre langue et une autre culture. (Günay, 2001, p. 12)

Il s'agit là, de penser désormais dans la langue cible. Cependant, il faudrait savoir que la possibilité de ceci est due à la familiarité avec la chose du texte. II s'agit d'une condition sine qua non pour traduire efficacement et objectivement. La possibilité d'objectivation que suppose ce lien à la chose permet de comprendre que l'acte créatif ne consiste pas à faire fi du sens. Puisqu'il dépend de la capacité à reconnaître et à retourner à la chose du texte. En ceci que «le retour à la chose, appelé par l'entreprise de restitution, ne pourrait s'effectuer, s'il n'incluait l'aveu d'une proximité, d'une complicité ou d'une familiarité de cette chose avec l'interprète. L'interprète, en restituant le sens de la chose, explicite en fait le sens qu'il prête à celle-ci. Mais ce sens prêté est un sens dû : c'est le sens de la chose» (Resweber, 1988, p. 26).

La langue étant le fait d'une influence environnementale, le texte que présente le traducteur reflète son nouvel environnement. Or, étant donné que «'l'aspiration à doter l'idée de perfection langagière d'une existence dans la réalité' est commune à toutes les langues [...]» (Gadamer, 1996, p. 463), son effectuation fait office de créativité. La 
raison de ce phénomène inhérent à la traduction pourrait être le fait que «l'autre monde, que l'on apprend ainsi à connaître, n'est justement pas un simple objet d'étude, de compétence et d'information. [Car, le traducteur] qui laisse venir à lui la tradition littéraire d'une langue étrangère de façon qu'elle se mette à lui parler n'entretient pas un rapport d'objectivité avec la langue comme telle [...]» (Gadamer, 1996, p. 466), mais avec la chose du texte - d'où le fait que ce qui importe en traduction soit «la qualité de l'entente sur quelque chose».

En outre, une créativité qui se veut objective implique un perfectionnement du dit. Ceci s'explique du point de vue herméneutique par le phénomène d'application, au sens gadamérien, qui préside dans cette acte. Le fait est que le traducteur traduit en fonction d'une double vision du monde : la sienne et celle de sa langue. Car, la compréhension qui l'autorise à traduire se fait sous fond d'application à soi de ce qui est compris. Selon Gadamer : «exercer ce comprendre, c'est déjà être requis par ce qui est dit, ce qui ne peut avoir lieu sans que l'on mette également en jeu'sa propre vision du monde et même sa propre vision de la langue'» (Gadamer, 1996, p. 466). Être créatif, c'est donc être capable de trouver effectivement ce qui pourrait perfectionner le sens à rendre. Sauf que cette acte créatif ne doit être en aucun cas le fait d'une extravagance ; il doit être fondé.

Remarquons que la créativité, tel que nous le cernons prémunit d'un atout pratique qui est d'ordre psychologique. Il se décline en deux prédispositions : la première est le fait de devoir assoupir le désir de traduire en se sentant déculpabilisé de l'idée de devoir trahir en rendant le sens autrement. La seconde est le fait de jouir de la liberté d'accomplir aisément sa tâche en fonction de la vision du monde de la langue d'arrivée et de sa propre vision de cette langue. En outre, d'un point de vue téléologique, le lecteur attend du traducteur qu'il soit le meilleur interprète du texte original. Finalité qu'on ne saurait atteindre sans être créatif. La cohérence herméneutique s'exprime ici dans l'idée du «mieux comprendre l'auteur qu'il ne s'est compris lui-même» - fait qui témoigne d'une compréhension parfaite (Schleiermacher, 1987, p. 108) - ou de la possibilité de «comprendre autrement» - fait qui témoigne d'une productibilité de la compréhension (Gadamer, 1996, p. 318). Ainsi, le fait d'être créatif est un gain pour la réceptivité de l'auteur et un avantage pour la fluidité du texte final auprès du lecteur. Cela étant, on peut comprendre pourquoi l'acte de traduire, tel que retenu par Le Robert, se conçoit comme la possibilité de «faire que ce qui était énoncé dans une langue le soit dans une autre, en tendant à l'équivalence de sens et de valeur des deux énoncés» (Robert, 1990). 
De fait, ce qui s'offre à la découverte lorsqu'on se livre à un engagement créatif, c'est I'universel que l'auteur exprime. Pour mieux saisir ceci, remarquons préalablement que tout traducteur qui prend sa tâche au sérieux est conscient du fait qu'«il n'existe pas de correspondances formelles, verbales ou structurelles, ni de correspondances sémantiques entre les langues» (Resweber, 1988, p. 124). Ce qui revient à dire que dès le vouloir traduire, il sait qu'il se voue, non pas à la traduction de la langue, mais de la valeur, de l'universel qui s'y trouve. Le fait est qu'il y a des expressions littéraires ou poétiques spécifiques aux différentes langues qui, du fait de leur spécificité culturelle, ne sont pas toujours adéquates à celle des langues cibles. La réplique métaphorique dans le cas de ces textes s'avère le plus souvent difficile. Nous observons, dans le cadre de la poésie par exemple, une union inséparable du sens et de la sonorité et/ou du signifié et du signifiant. En outre, il s'est avéré, dans le cadre des œuvres philosophiques, par exemple, qu'on fasse face à une difficulté d'un autre ordre qui

surgit au plan même du découpage des champs sémantiques qui s'avèrent non exactement superposables d'une langue à l'autre. Non seulement, précise Ricœur, les champs sémantiques ne se superposent pas, les syntaxes ne sont pas équivalentes, les tournures de phrases ne véhiculent pas les mêmes héritages culturels; et que dire des connotations à demi muettes qui surchargent les dénotations les mieux cernées du vocabulaire d'origine et qui flottent en quelque sorte entre les lignes, les phrases, les séquences courtes ou longues. (Ricœur, 2004, pp. 12-13)

À cet effet, Resweber fait remarqué que

C'est ce que veut sans doute signifier le constat, maintes fois répété, qu'une langue est intraduisible. Mais la résistance linguistique à la traduction se double d'une résistance culturelle qui devient dominante, lorsqu'il s'agit des textes littéraires ou poétiques. La langue fait résonner une culture, car elle renvoie à un système symbolique de valeurs qui en déterminent les connotations. C'est néanmoins cette seconde résistance qui rend possible la traduction, dans la mesure où elle comporte des universaux culturels, exprimant les articulations du corps ou de la parole, du désir et du sexe, de l'amour et de la mort. Ce sont ces universaux qui, par delà les correspondances des codes linguistiques, fournissent au traducteur les pôles herméneutiques autour desquels s'organisent les unités de traduction. (Resweber , 1988, p. 124) 
Alors que l'alternative de la fidélité textuelle se présente comme un chemin truffé de difficulté, celle de la créativité ouvre à la découverte des possibilités de commutation. Celle-ci est, selon Le Robert une «opération par laquelle on substitue les uns aux autres certains éléments phoniques ou sémantiques, permettant ainsi de constituer des paradigmes et de dégager les distinctions linguistiques pertinentes» (Robert, 1990). C'est de là qu'on réalise la possibilité de sortir du drame pour réaliser le défi de la bonne traduction (Ricœur, 2004, p. 11) par l'entremettre des valeurs universelles qui se dégagent dans la langue de départ. Ce désir même de traduire, de porter et de partager cet universel à l'autre, tel que le montre Antoine Berman dans L'épreuve de l'étranger (Berman, 1984), s'accompagne du souci d'être reçu dans l'autre langue que seule la créativité permet. C'est pourquoi la bonne traduction est celle qui se contente d'une écriture adéquate à la langue cible à telle enseigne que l'on pourrait confondre le traducteur à l'auteur du texte ou encore le texte d'arrivée à un texte originaire. Une écriture adéquate à la langue cible revient à dire que le traducteur est un auteur. Tout simplement, "parce qu'il invente lui-même les unités de traductions, représentant des segments variables qui se correspondent par commutation» (Resweber, 1988, p. 124). En outre, le caractère fonctionnel de la traduction et donc la spécificité des idiomes, fait que la fidélité ne se pose plus comme un souci. Michon écrit :

Si l'on prend le texte traduit comme un point de départ et non comme un point d'arrivée, la question de la fidélité de la traduction se transforme radicalement. Le problème n'est plus de faire en sorte que le texte soit le plus fidèle possible aux signifiés de celui que l'on veut traduire, mais plutôt d'écrire un texte qui arrive à faire dans sa langue-culture ce que le texte traduit fait dans la sienne. (Michon, 2000, p. 49)

On comprend que le souci d'une traduction ne devrait pas être de dire la même chose, pour prétendre à une bonne traduction, mais de trouver des équivalences de sens. Ce qui soulagerait du désir d'une traduction parfaite qui serait utopique d'après Ricœur. Il estime d'ailleurs que l'acte de traduire consiste à faire le deuil de la traduction absolue et par ailleurs le bonheur de la traduction. De ce drame heureux, il note :

Et c'est ce deuil de la traduction absolue qui fait le bonheur de traduire. Le bonheur de traduire est un gain lorsque, attaché à la perte de l'absolu langagier, il accepte l'écart entre l'adéquation et l'équivalence, l'équivalence sans adéquation. Là est son bonheur. En avouant et en assumant 
l'irréductibilité de la paire du propre et de l'étranger, le traducteur trouve sa récompense dans la reconnaissance du statut indépassable de dialogicité de l'acte de traduire comme l'horizon raisonnable du désir de traduire. (Ricœur, 2004, p. 19)

L'équivalence sans adéquation reconnue comme étant le propre de toute traduction réussite est la résultante de l'opérativité de la créativité. Le tout se jouant dans la commutation des équivalences sémantiques et stylistiques pour réexprimer l'universel, nous pouvons dire sans ambages que la traduction consiste à re-créer un énoncé dans une autre langue, ou de trouver les équivalents de cet énoncé dans la langue d'arrivée. Il est à noter que le rapport de conformité dont il est ici question, est strictement lié à la valeur. Ce qui suppose que le système linguistique de la langue de départ ne saurait être identique à celle de la langue d'arrivée. De plus, étant donné que le phénomène de traduction met en jeu une lecture critique spécifique, la tâche du traducteur dans son engagement créatif est de parvenir à une équivalence qui se pose comme garant d'une traduction réussite. Puisque l'adéquation ou l'identité s'avère difficile à atteindre, nous devrons retenir qu'«une bonne traduction ne peut viser qu'à une équivalence présumée, non fondée dans une identité de sens démontrable. Une équivalence sans identité. Cette équivalence ne peut être que travaillée, présumée» (Ricœur, 2004, p. 40). La preuve de cette présomption est le fait qu'en toute re-traduction, extralinguistique ou intralinguistique telle qu'on observe pour les grandes œuvres, on se rend compte davantage de l'impossibilité d'une traduction parfaite. Tout ce qui est finalement avoué, c'est la différence indépassable entre le propre et l'étranger.

La créativité permet finalement que tout ce qui est étranger dans l'autre langue ne soit pas étrange pour le lecteur du texte dans la langue d'arrivée. En tant qu'elle est aussi heuristiquement fondée, cette créativité consiste essentiellement à la découverte des équivalences qui pourront satisfaire le désir d'appropriation du lecteur. En définitive, on peut dire avec Günay que «l'opération de traduire est un acte d'écrire un autre texte dans la langue d'arrivée à partir du texte de la langue de départ, par le traducteur ayant le souci de trouver les équivalents, dans la langue d'arrivée, des unités linguistiques du texte dans la langue de départ. En conclusion, la traduction consiste à passer d'un code à un autre, dans le processus de communication» (Günay, 2001, p. 19). Il s'avère qu'au détriment d'une fidélité textuelle, la créativité convie à une équivalence sans adéquation, entre le texte de départ et celui d'arrivée, au terme du processus de traduction. Cependant, il s'agit d'une posture tout à fait mitigée en traductologie. 


\section{La créativité, perte de spécificité ou abri du sens}

II nous semble, d'après l'analyse qui suit, que la fidélité textuelle soit renvoyée aux calendes grecques. Mais en réalité, nous ne devons pas oublier qu'elle est participante d'une dialectique pratique qui trouve son exigence qu'en situation. «[L]a situation est ici celle d'une entente particulièrement laborieuse, au cours de laquelle on reconnaît que la distance qui sépare ce que veut dire l'autre de ce qu'on veut dire soi-même ne peut en définitive être abolie» (Gadamer, 1996, p. 408). Cette impuissance en situation a donné lieu de réduire la traduction, si l'on s'en tient à la déclaration de Joseph Conrad à Davray, à un exercice de perfectionnement d'un texte d'une langue à l'autre : "Je suis persuadé que vous donnerez à mon œuvre (qui me semble, quand je la regarde, rien que cendres et poussières) non seulement un corps nouveau, mais aussi le souffle qui la rendra vivante» (West, 1996, p. 45). Il s'agit là d'une volonté de concevoir la créativité inhérente en traduction comme quelque chose visant à donner, délibérément, un autre souffle au texte d'arrivée que celui de l'auteur source. C'est dire qu'ici, l'acte créatif devrait sa nécessité du soupçon. Or, il ne s'agit pas de cela, car, notions-nous, tout l'effort dès le vouloir traduire est d'être fidèle. Ce n'est qu'en situation, animer du «désir de traduire» (Ricœur, 2004, pp. 38-39), que ce vœu de fidélité, au sens où il engage plus de littéralité, se trouve relativisée. Mais ici, le fait de donner à la traduction une vocation prédéfinie exaspère le «consentement à la perte». Ce qui donne lieu à revoir le statut de la fidélité textuelle.

À y réfléchir, on pourra se rendre compte qu'il s'agit d'une reconsidération à défaut d'une créativité excessive. Aussi, peut-on observer chez des auteurs une résistance quant au phénomène d'«interprétation infinie» qui s'oppose à tout littéralisme. De Bori (1987) à Eco (1992) on arrive à penser aux «limites de l'interprétation». II n'est plus question d'interpréter pour signifier davantage, car ce serait abuser de la signifiance en désavouant toute propension à la fidélité textuelle. La créativité qui s'y oppose est perçue comme l'effet d'un refus d'imprégnation de la culture source en tant que possibilité que promeut la traduction adéquate. Pour Eco, cette imprégnation déterminerait la compréhension du texte et sa traduction. «Pour comprendre un texte - a fortiori pour le traduire - il faut faire une hypothèse sur le monde possible qu'il représente [...] s'appuyer sur des conjonctures [...] c'est seulement après avoir élaboré une conjoncture plausible que le traducteur peut commencer à faire passer le texte d'une langue à l'autre» (Eco, 2006, p. 51). Mais, la négation de la productibilité est inopérante face aux limites d'ordre linguistique entre idiome. Eco en est conscient : 
«Traduire signifie parfois se rebeller contre sa propre langue quand elle introduit des effets de sens qui n'étaient pas entendus dans la langue originale» (Eco, 2006, p. 139). Aussi eut-il envisagé de restreindre la possibilité d'aider le texte : «ll faut toutefois résister à la tentation de trop aider le texte, en se substituant presque à l'auteur» (Eco, 2006, p. 126). Mais on pourra observer dans la progression de ses idées qu'il en arrive à établir la fidélité textuelle comme gage d'une bonne traduction. Il écrit : «certaines traductions enrichissent à merveille la langue de destination et réussissent parfois de manière très heureuse, à en dire plus (car plus riches en suggestions) que les originaux. [...] Une traduction qui arrive à 'en dire plus' pourra être une excellente œuvre en soi, mais pas une bonne traduction» (Eco, 2006, p. 139). Toutefois, il est impossible d'établir le critère absolu de bonne traduction, car «pour qu'un tel critère soit disponible, il faudrait qu'on puisse comparer le texte de départ et le texte d'arrivée à un troisième texte qui serait porteur du sens identique supposé circuler du premier au second. La même chose dite de part et d'autre» (Ricœur, 2004, p. 39).

II s'avère qu'en toute traduction, le désir de traduire l'emporte sur le dilemme. II s'agit déjà d'une tâche pas tout à fait aisée. On devrait se rendre compte que, le fait de devoir considérer la créativité comme simple alternative à la fidélité n'est que le reflet d'une réalité de «l'entre-deux» (Wecksteen, 2011) ou du fait d'être un «agent double» (Mariaule et Wecksteen, 2011) qui ne devrait pas impacter su le but du traducteur. S'il faut concevoir la tâche comme un projet créatif, ce qui convient au traducteur, c'est une «esthétique de l'écoute et de la réceptivité» (Ladmiral, 1994, p. 110) qui lui permettrait de s'approprier le sens à rendre et d'assumer la condition de restitution qui se détermine par une productibilité particulière. La principale raison, la plus évidente d'ailleurs, est qu'entre deux cultures, il y a des imperfections qui oblige le traducteur à opérer un choix. Dans la plupart des cas le souci de vraiment transmettre l'oriente vers une volonté de parfaire. Michon relève ici l'ambigüité de cette impasse :

Étant donné l'imperfection inévitable de toute entreprise de translation d'une langue à une autre, le traducteur n'aurait d'autre choix qu'entre deux insuffisances : soit privilégier la langue d'arrivée en facilitant la lecture mais en donnant une impression trompeuse de transparence, soit privilégier la langue de départ par une traduction littérale qui tente de restituer l'original mais qui viole la langue d'arrivée en produisant des obscurités. (Michon, 2000, p. 48) 
Mais on ne s'aurait demeurer dans cette impasse, puis qu'il faudrait prendre en compte un paramètre crucial, celui de l'historicité de la compréhension propre à la subjectivité du traducteur. On a généralement deux cas de figure qui contraignent le traducteur à devoir faire fi de l'alternative de la fidélité textuelle pour celle de la créativité, malgré toute la ferme volonté de s'abstenir de dire plus. La première se comprend à partir de la célèbre formule herméneutique du «mieux comprendre l'auteur qu'il ne s'est compris lui-même». La seconde se comprend à partir de la potentialité de chaque signe : la signifiance. Celle-ci traduit une capacité à signifier davantage qui invite à l'interprétation, et donc à une ouverture à des éventualités. Cependant, on observe des traces d'intraduisibilité dans des textes qui témoignent, tout de même, d'une volonté de respect à l'endroit du texte originel. De là, on pourrait noter une restriction de la traduction volontaire et un refus de traduction part souci de pudeur.

Tout compte fait, nous pensons que la liberté du traducteur s'arrête là où la fidélité au sens du texte original est menacée. Le fait de vouloir à tout prix insuffler dans l'œuvre traduite l'esprit d'une langue qui lui est étrangère ou de vouloir à tout prix métamorphoser la langue source pourrait rimer à autre chose qu'une volonté de transmission de sens. Au-delà d'une possibilité d'enrichissement de l'une des langues ou des deux, une fidélité textuelle peut se traduit en une volonté hégémonique et une créativité en une volonté de préservation d'une spécificité ; tout ceci au détriment d'une ferme volonté de rendre un sens. Une expérience de la traduction qui se veut soucieuse du sens à rendre ne saurait clivée radicalement vers l'un des pôles, fidélité textuelle et créativité, ou oscillée entre les deux. Ricœur rappelle ceci :

Les traducteurs le savent bien : ce sont des textes, non des phrases, non des mots, que veulent traduire nos textes. Et les textes à leur tour font partie d'ensembles culturels à travers lesquels s'expriment des visions du monde différentes, qui d'ailleurs peuvent s'affronter à l'intérieur du même système élémentaire de découpage phonologique, lexical, syntaxique, au point de faire de ce qu'on appelle la culture nationale au communautaire un réseau de visions du monde en compétition occulte ou ouverte [...]. (Ricœur, 2004, p. 55)

C'est dire que ce qui finalement doit prévaloir, c'est le sens rendu. Puisque ce qui rendra valide la traduction auprès de l'original, c'est la qualité de l'entente sur la chose. Cependant, ce qui fait cette qualité de l'entente sur la chose, c'est le fait qu'on puisse 
comprendre l'original du point de vue de la traduction. Qu'en lisant le texte traduit dans l'autre langue qu'on comprenne comme si on n'était dans la langue de l'original de tel sorte que l'on ne se rende pas compte qu'on a changé de langue. Le lecteur devrait tout simplement comprendre son texte et dialoguer avec lui. Tel devrait être le souci de toute traduction : faire que l'auteur puisse parler au lecteur et qu'ils se comprennent comme s'il partageait la même langue ; car «quand on se comprend, on ne traduit pas, on parle» (Gadamer, 1996, p. 406).

Nous pensons que l'idée de fidélité dans cette tâche de traduction devrait être une exigence liée au sens et que toute créativité devrait être une initiative inventive visant à rendre ce sens. Si vraiment, par le souhait de fidélité textuelle l'on voudrait goûter, autant que possible, aux mêmes sensations que l'auteur, c'est-à-dire, se comporter comme si nous étions dans sa peau, ce qui pourrait le permettre serait la méthode divinatoire'. Sauf que celle-ci est inopérante en traduction. Schleiermacher (1999) I'un des promoteurs de cette méthode le reconnait fort bien lorsqu'il note : «qu'il est difficile d'atteindre le but véritable de toute traduction, goûter si possible sans le moindre travestissement les œuvres étrangères si l'on obéit à une méthode qui veut absolument insuffler dans l'œuvre traduite l'esprit d'une langue qui lui est étrangère» (Marc de Launay, 2006, p. 105).

II n'y a, en vérité, rien qui puisse permettre au traducteur de pouvoir concrétiser ce souhait de la fidélité textuelle qui, positivement, I'habite en tant qu'émanation d'un désir de traduction parfaite. Même la contemporanéité, la familiarité que le traducteur pourrait avoir avec la vie et les sentiments de l'auteur ne concourrait pas à la réalisation de ce souhait. Dans la même veine, Gadamer argue :

Il est indubitable que, quelle que soit la familiarité du traducteur avec la vie et les sentiments de son auteur, la traduction d'un texte n'est pas la simple résurrection du processus psychologique originel de sa rédaction, mais une reprise (Nachbildung), guidée par la compréhension de ce qui est dit. Personne ne peut douter qu'il s'agit ici d'une interprétation et non d'une simple co-opération (Mitvollzug). (Gadamer, 1996, p. 407)

Le caractère interprétatif de la traduction déterminerait absolument sa mise en marge, du point de vue pratique, de la fidélité textuelle pour un attrait à l'équivalence du sens.

1 «La méthode divinatoire est celle dans laquelle, en se transformant pour ainsi dire soi-même en l'autre, on cherche à saisir immédiatement l'individuel» (Schleiermacher, 1987, p. 150). 
En dernière analyse, il faudrait reconnaître que le substrat de toute traduction est le sens. La traduction ne consistant en une reproductibilité mais une productibilité, le traducteur à la tâche de faire naître le sens dans la langue hôte. Ainsi, au-delà de l'entredeux de la fidélité textuelle et de la créativité, le critère absolu d'une bonne traduction «serait le même sens, écrit quelque part, au-dessus et entre le texte d'origine et le texte d'arrivée» (Ricœur, 2004, p. 60) et non celui d'une fidélité textuelle utopique ou d'une créativité ayant un contenue relatif. Bien que la sémiotique contemporaine attire l'attention sur l'unité du sens et du son, du signifier et du signifiant, il serait risqué de sortir de l'abri confortable de l'équivalence du sens.

Toute traduction doit être au service du sens. Ce service est jugé honorable quand le traducteur et le lecteur font preuve de satisfecit. Si l'on parvient réellement au bout du compte à une fidélité textuelle tout en rendant le sens ce serait l'idéal. Le malaise de l'entre-deux auquel se confronte la traduction a toujours eu à donner raison à la perspective créative depuis l'antiquité. Cicéron quand il s'y confronta, prôna la restitution des idées (du sens) plutôt que de recourir au littéralisme : «en conservant toutes les beautés qu'ils offrent, c'est-à-dire, les pensées, les figures, l'ordre des choses, et en ne m'attachant aux expressions qu'autant qu'elles peuvent s'accommoder aux usages de notre langue. Si toutes ne se trouvent pas traduites, je me suis efforcé, du moins, d'en rendre l'esprit» (Cicéron cité par Jérôme, 1837, p. 150). II n'est point question d'avoir une posture déterminer en face de ce phénomène tout à fait naturel de l'entre-deux - sous peine de s'enrayer -, mais de laisser s'opérer la dialectique de l'identité et de la différence, du propre et de l'étranger le regard fixé sur ce qui fait sens. Puisqu'au final, «tant que l'on ne sent pas l'étrangeté, mais l'étranger, la traduction a rempli son but suprême ; mais là où l'étrangeté apparaît en elle-même et obscurcit peut-être même l'étranger, alors le traducteur trahit qu'il n'est pas à la hauteur de son original» (Von Humboldt, 2000, p. 39).

Quoiqu'il en soit, cet entre-deux de la fidélité textuelle et de la créativité dans lequel la traduction ce situe convie à faire prévaloir la capacité d'intégration du sens comme relevant d'une prédisposition de la part du traducteur et de sa possibilité d'établir des équivalents au sien de la langue hôte. Ce qui nous ramène à deux choses qui devrait être pris en compte en traductologie : une «esthétique de la réception» (Jauss, 1978) et une «hospitalité langagière» (Ricœur, 2004, p. 43). En effet, il est question qu'en toute traduction, qu'on puisse être capable de transcender et même de relativiser la tension de l'entre-deux pour l'essentiel (le sens). La spirale de la retraduction que reconnait 
Ricœur, par exemple, tient sa validité qu'il y a cet essentiel qui l'autorise. Chaque traduction possible trouverait sa validité épistémologique suivant les prérogatives d'une herméneutique littéraire. Souvenons-nous que l'herméneutique littéraire rend «compte du caractère proprement esthétique des œuvres» (Jauss, 1988). Ce faisant, on pourrait, à partir de ses prérogatives, établir en quoi, eu égard à l'enclin de devoir «dire la même chose autrement» (Ricœur, 2004, p. 45; Eco, 2006), l'autrement dit est un dit légitime, uniquement distinct par une esthéticité propre. Mais celle-ci est tributaire d'une esthétique de la réception. Le fait est qu'en accordant un «primat herméneutique» au traducteur, on présume qu'il fasse une juste lecture matérialisée par la pertinence des éléments qu'il choisit délibérément comme pouvant porter le sens du texte de départ. À la vérité, «il faut lui accorder un primat d'accès méthodologique, parce qu'il est plus facilement objectivable» (Jauss cité par Kalinowski, 1997, p. 158). Ceci dans la mesure où on pourrait trouver une explication de ses choix, car «traduire nous contraint non pas seulement à trouver un mot mais à reconstruire le sens authentique du texte dans un horizon linguistique tout à fait nouveau ; une traduction véritable implique toujours une compréhension qu'on peut expliquer» (Gadamer, 1982, p. 45). Ces propos de Gadamer permettent de comprendre qu'en tout «autrement dit» il y a une raison qui y préside. Toutefois, si l'on prend en compte le caractère transitoire de chaque traduction, nous verrons que la diversité des raisons qui pourraient être présentées se subsume dans ce que Ricœur nomme «hospitalité langagière».

De fait, le paradoxe de la différence nous oblige à consentir à l'accueil de l'autre langue. Ceci ne se fait que suivant une «dialectique pratique» qui commande une démarche hospitalière où il est question de braver l'impossibilité d'une adéquation par la possibilité d'une équivalence. C'est donc cette «juste distance dans le travail de construction d'éléments comparables qui prend la forme d'une'hospitalité langagière'» (Díez, 2014, p. 94). Celle-ci se présente comme la «forme d'accueil d'un texte dans une langue et dans une culture qui ne sont pas celles d'origine» (Díez, 2014, p. 95). Le traducteur a conscience de son travail d'accueil et d'intégration du sens dans sa langue. En tant qu'hôte sa vocation première est l'intégration : «Intégrer est soumettre l'autre à ma loi, exiger sa métamorphose, sa transformation, c'est-à-dire, exercer d'une certaine manière une violence» (Díez, 2014, p. 96). Ainsi, toute traduction est avant tout respect de l'idiome de la langue d'accueil, acceptation de la distance entre l'esprit de ce qui a été dit et celui de son interprétation et assomption de la responsabilité du surcroît de clarté apporter au texte original. 
L'hospitalité langagière présumée qui justifie l'acte même de traduire n'est envisagée que sur fond d'un engagement créatif et non d'une fidélité textuelle. La créativité aujourd'hui reconnue comme processus au cœur de la traduction n'est pas une alternative. Le traducteur ne peut qu'être que créatif, car l'essence même de la traduction est d'être créative.

\section{Conclusion}

À partir de la question «qu'est-ce qui fait la valeur d'une traduction ?», cet article a tenté d'analyser par le biais de l'herméneutique le concept de créativité qui y a cours pour étayer le fait que la traduction est essentiellement un projet créatif. Récusant par ces étais le fait que la créativité soit perçue comme une simple alternative en face de celle de la fidélité textuelle, nous montrions dans un premier temps qu'elle est intrinsèquement féconde. En ceci que le traducteur qui se soumet à une «esthétique de la réception» parvient à s'entendre sur la chose en question. La qualité de cette entente se traduit dans sa capacité de rendre le sens selon un choix délibéré de sa vision de la langue hôte qui contribue pour autant à l'effet de porter le sens à son plus haut et intelligible expression. Nous y voyons une expression de la créativité comme inhérente au traduire. Fonder sur le fait d'une «hospitalité langagière» on peut se rendre compte que la traduction trouve son accomplissement dans une équivalence de sens et de valeur des deux énoncés que dans une adéquation ou identité qui correspondrait à une fidélité textuelle. Bien que le manque de fidélité textuelle peut être perçu comme une entrave à la spécificité esthétique de l'auteur, ceci n'étant par le fait d'une volonté propre du traducteur, mais de l'effet de la différence idiomatique, nous pensons que l'acte créatif qui permet de satisfaire le noble désir de traduire devrait pas être perçu comme une trahison ou comme une simple alternative, mais comme l'essence même de la traduction. Aussi, l'appropriation du même sens que celui du texte original par des lecteurs, indépendamment de leur rapport distinct à la langue propre ou étrangère, témoigne du fait que la condition linguistique de la pensée ne représente pas seulement une condition limitative, mais toujours déjà la condition positive de son déploiement. Tel est ce que nous retenons de ce concept de traduction à partir d'une réflexivité herméneutique, ce qui ne traduit pas pour autant une approche exhaustive de la question. 
Évaluation : Évaluation anonyme par des pairs extérieurs.

Conflit d'intérêts : L'auteur n'a aucun conflit d'intérêts à déclarer.

Subvention : L'auteur n'a reçu aucun soutien financier pour ce travail.

Peer-review: Externally peer-reviewed.

Conflict of Interest: The author has no conflict of interest to declare.

Grant Support: The author declared that this study has received no financial support.

\section{Bibliographie}

Allouch, J. (1984). Lettre pour lettre : transcrire, traduire, translittérer. Erès : Toulouse.

Berman, A. (1995). L'épreuve de l'étranger. Culture et traduction dans l'Allemagne romantique. Paris : Gallimard.

Bori, P. C. (1987). L'interprétation infinie. Paris : Cerf.

Díez, F. (2014). L'hospitalité langagière. Paul Ricœur et la question de la traduction. Esprit. Paris : Editions Esprit. 402(02), 87-98.

Eco, U. (2006). Dire presque la même chose. Expériences de traductions. Paris : Grasset et Fasquelle.

Eco, U. (1992). Les limites de l'interprétation. Paris : Grasset.

Gadamer, H.-G. (1982). L'art de comprendre. Écrits I. Herméneutique et tradition philosophique. Paris : Aubier.

Gadamer, H.-G. (1995). Langage et vérité. Paris : Gallimard.

Gadamer, H.-G. (1996). Vérité et méthode. Les grandes lignes d'une herméneutique philosophique. Paris : Seuil.

Günay, D. (2001). Le traducteur, un co-auteur. Le Français Dans Le Monde. Paris : Hachette, n 314.

Heidegger, M. (1937). Questions / et II. Paris : Gallimard.

Hénault, A. (1979). Les enjeux de la sémiotique. Paris : PUF.

Jauss, H.-R. (1978). Pour une esthétique de la réception. Paris : Gallimard.

Jauss, H-R. (1988). Pour une herméneutique littéraire. Paris : Gallimard.

Jérôme, (1837). Lettres. Tome III, Librairie catholique de Perisse Frères. Web. 19 décembre 2019. <http://www. libreius.net/livres/lettres_de_saint_Jerome_(tome_3)_000000531.pdf>.

Kalinowski, I. (1997). Hans-Robert Jauss et l'esthétique de la réception. Revue germanique internationale, 8, 151172.

Ladmiral, J.-R. (2013). La question de la littérature et les traductions de Freud. In S. Schwerter \& J. Dick. (Eds.). Traduire:transmettre ou trahir?, Edition de la Maison des Sciences de I'homme.

Ladmiral, J.-R. (1994). Traduire : théorèmes pour la traduction. Paris : Gallimard.

Marc de Launay, (2006). Qu'est-ce que traduire ? Paris : Vrin.

Mariaule, M., \& Wecksteen. C. (2011). De Janus à 15 Ménechme : portrait du traducteur en agent double. In C.

Wecksteen (Ed.), Le double en traduction ou l'(impossible) entre-deux. Arras : APU, 29-48.

Meschonnic, H. (1973). Pour la poétique Il, Épistémologie de l'écriture, Poétique de la traduction. Paris : Gallimard. 
Michon, P. (2000). Traduire. In P. Michon. Poétique d'une anti-anthropologie. L'herméneutique de Gadamer (pp. 4564). Paris : Vrin.

Mounin, G. (1986). Les Problèmes Théoriques de la Traduction. Paris : Gallimard.

Resweber, J.-P. (1988) Qu'est-ce qu'interpréter ? Essai sur les fondements de l'herméneutique. Paris : Cerf.

Ricœur, P. (2004). Sur la traduction. Paris : Bayard.

Robert, P. (1990). Le Petit Robert: Dictionnaire alphabétique et analogique de la langue française. Paris : Le Robert.

Schleiermacher, F. (1999). Des différentes méthodes de traduire. Paris : Seuil.

Schleiermacher, F. (1987). Herméneutique. Pour une logique du discours individuel. Paris : Cerf.

Steiner, G. (1998). Après Babel. Une poétique du dire et de la traduction. Paris : Albin Michel.

Toury, G. (2004). Los estudios descriptivos de traducción y más allá. Metodología de la investigación en estudios de traducción. Madrid : Cátedra.

Von Humboldt, W. (2000). Sur le caractère national des langues et autres écrits sur le langage. Paris : Seuil.

Wecksteen, C. (2011). (Ed.). Le double en traduction ou l'(impossible) entre-deux. Arras : APU.

West, R. (1996). Conrad and Gide. Translation, Transference and Intertextuality. Amsterdam : Rodopi. 
